\title{
Kappa-Free Light Chains in CSF Predict Early Multiple Sclerosis Disease Activity
}

Klaus Berek, MD, Gabriel Bsteh, MD, PhD, Michael Auer, MD, PhD, Franziska Di Pauli, MD,PhD, Astrid Grams, MD, Dejan Milosavljevic, BSc, MSc, Paulina Poskaite, MD, Christine Schnabl, MSc, Sebastian Wurth, MD, Anne Zinganell, MD, Thomas Berger, MD, MSc, Janette Walde, PhD, Florian Deisenhammer, MD, MSc, and Harald Hegen, PD, MD, PhD

Neurol Neuroimmunol Neuroinflamm 2021;8:e1005. doi:10.1212/NXI.0000000000001005

\section{Abstract}

\section{Objective}

To investigate whether $\kappa$-free light chain ( $\kappa$-FLC) index predicts multiple sclerosis (MS) disease activity independent of demographics, clinical characteristics, and MRI findings.

\section{Methods}

Patients with early MS who had CSF and serum sampling at disease onset were followed for 4 years. At baseline, age, sex, type of symptoms, corticosteroid treatment, and number of T2 hyperintense (T2L) and contrast-enhancing T1 lesions (CELs) on MRI were determined. During follow-up, the occurrence of a second clinical attack and start of disease-modifying therapy (DMT) were registered. $\kappa$-FLCs were measured by nephelometry, and $\kappa$-FLC index calculated as $[\mathrm{CSF} \kappa$-FLC/serum $\kappa$-FLC]/albumin quotient.

\section{Results}

A total of 88 patients at a mean age of $33 \pm 10$ years and female predominance of $68 \%$ were included; 38 (43\%) patients experienced a second clinical attack during follow-up. In multivariate Cox regression analysis adjusting for age, sex, T2L, CEL, disease and follow-up duration, administration of corticosteroids at baseline and DMT during follow-up revealed that $\kappa$-FLC index predicts time to second clinical attack. Patients with $\kappa$-FLC index $>100$ (median value 147) at baseline had a twice as high probability for a second clinical attack within 12 months than patients with low $\mathrm{k}-\mathrm{FLC}$ index (median 28); within 24 months, the chance in patients with high $\kappa$-FLC index was 4 times as high as in patients with low $\kappa$-FLC index. The median time to second attack was 11 months in patients with high $\kappa$-FLC index whereas 36 months in those with low $\kappa$-FLC index.

\section{Conclusion}

High $\kappa$-FLC index predicts early MS disease activity.

\section{Classification of Evidence}

This study provides Class II evidence that in patients with early MS, high $\kappa$-FLC index is an independent risk factor for early second clinical attack.

\author{
Correspondence \\ Dr. Hegen \\ harald.hegen@i-med.ac.at
}

\section{MORE ONLINE}

(III) Class of Evidence

Criteria for rating

therapeutic and diagnostic studies

NPub.org/coe

\footnotetext{
From the Department of Neurology (K.B., M.A., F.D.P., A.Z., F.D., H.H.), Medical University of Innsbruck; Department of Neurology (G.B., T.B.), Medical University of Vienna; Department of Neuroradiology (A.G., P.P.), Medical University of Innsbruck; FH Campus Wien (D.M., C.S.), University of Applied Sciences, Vienna; Department of Neurology (S.W.), Medical University of Graz; and Department of Statistics (J.W.), Faculty of Economics and Statistics, University of Innsbruck, Austria.

Go to Neurology.org/NN for full disclosures. Funding information is provided at the end of the article. 


\section{Glossary}

CDMS = clinically definite multiple sclerosis; $\mathbf{C E L}=$ contrast-enhancing T1 lesion; DMT = disease-modifying therapy; EDSS = Expanded Disability Status Scale; $\boldsymbol{\kappa}$-FLC $=\kappa$-free light chain; $\mathbf{M S}=$ multiple sclerosis; $\mathbf{O C B}=$ oligoclonal band; $\mathbf{T} 2 \mathbf{L}=$ hyperintense T2 lesion; WBC = white blood cell.

Multiple sclerosis (MS) is a chronic inflammatory immunemediated neurologic disease that mainly affects young adults and bears the risk of physical and cognitive disability. ${ }^{1}$ In the last decades, an increasing number of disease-modifying therapies (DMTs) were proven to reduce the number of relapses, accumulation of disability, and brain MRI activity in relapsing MS. ${ }^{2}$ Current treatment concepts recognize the importance of early treatment and plead toward suppressing disease activity below the level of detectability. ${ }^{3}$

However, the interindividual courses of MS are extremely variable, ${ }^{4}$ and weighing benefits vs risks of certain DMT has become one of the main challenges for neurologists counseling patients with MS. ${ }^{5}$ Since criteria guiding decisions when to start treatment in early MS and, in case, whether choosing a moderately or a highly efficacious DMT, are still controversially debated, there is an urgent need for biomarkers to predict disease activity. ${ }^{5,6}$ So far, the number of brain MRI lesions and the presence of oligoclonal bands (OCBs) in the CSF imply some prognostic value and are widely accepted.?

Free light chains (FLCs) in the CSF are an emerging biomarker in MS that showed high diagnostic accuracy and significant methodological advantages over detection of OCB. ${ }^{8,9}$ Although there is some evidence that FLCs have also prognostic value, ${ }^{10,11}$ it is still unclear whether this holds true after adjusting for other prognostic factors. The objective of this study was to investigate whether $\kappa$ - and $\lambda$-FLC index predict disease activity in patients with early MS independent of demographics, clinical, and MRI characteristics.

\section{Methods}

\section{Study Design}

This study included patients of the MS clinic of the Department of Neurology, Medical University of Innsbruck, who had a first demyelinating event of the CNS, had CSF and serum collection for routine diagnostic purposes at disease onset, and received the diagnosis of clinically isolated syndrome or MS according to the McDonald criteria 2017..$^{12}$ Patients were prospectively followed over a period of 3-4 years.

At baseline, demographic characteristics and clinical and paraclinical variables were assessed. Demographics included sex and age. Clinical variables comprised disease duration (time between symptom onset and lumbar puncture), type of symptoms (monofocal vs multifocal syndrome; affection of the optic nerve, brainstem/cerebellum, spinal cord, or brain area of other topography), and use of corticosteroid treatment. Paraclinical variables were number of hyperintense lesions on T2-weighted MRI (T2L), number of contrastenhancing lesions on T1-weighted MRI (CEL), and main CSF findings including OCB status.

During follow-up, the confirmed occurrence of a second clinical attack (i.e., conversion to clinically definite MS [CDMS $]$ ) and start of DMT were registered. Clinical visits were arranged at the treating physician's discretion, at least once a year. At each visit, disability status was assessed by the Expanded Disability Status Scale (EDSS). ${ }^{13}$

\section{MRI}

MRI was performed for diagnostic purposes. T2-weighted and gadolinium-enhanced T1-weighted MRI scans of the brain (and if available of the spinal cord) were obtained. Contiguous, axial, maximal 5-mm-thick slices at a field strength of $1.5 \mathrm{~T}$ or $3 \mathrm{~T}$ were acquired. MRI analysis was performed centrally at the Department of Neuroradiology, Medical University of Innsbruck, by experienced raters blinded for any specific clinical information except referral for the suspected diagnosis of MS.

\section{CSF Analysis}

Routine CSF analysis was performed at the Neuroimmunology Laboratory, Department of Neurology, Medical University of Innsbruck. CSF white blood cell (WBC) and red blood cell counts were determined in a Fuchs-Rosenthal chamber, which has a volume of $3.2 \mu \mathrm{L} .^{14}$ Division by 3.2 allowed reporting of cell counts per $\mu \mathrm{L}$ according to the International System of units (SI). Albumin in CSF and serum were measured by nephelometry (Beckman Coulter $\mathrm{GmbH}$, Brea, CA) as previously reported. ${ }^{15}$ The albumin quotient $\left(\mathrm{Q}_{\mathrm{alb}}\right)$ was calculated as the ratio of CSF albumin/serum albumin. ${ }^{14}$ Detection of OCB was performed by isoelectric focusing and subsequent immunoblotting using IgG-specific antibody staining as previously described. ${ }^{16}$ OCB patterns were evaluated by experienced raters and classified as negative (patterns I, IV, and V) or positive (patterns II and III). ${ }^{17} \mathrm{~A}$ cutoff $>2$ CSF-restricted bands was used to define OCB positivity. ${ }^{16}$

\section{FLC Assay and Calculation of Intrathecal FLC Synthesis}

$\kappa$ - and $\lambda$-FLC concentrations in CSF and serum samples were analyzed by nephelometry using Behring ProSpec with the serum FLC immunoassay (N Latex FLC kappa and N Latex FLC lambda assay, Siemens, Erlangen, Germany) according 
Table 1 Demographic, Clinical, MRI, and CSF Characteristics

\begin{tabular}{|c|c|c|c|c|c|c|}
\hline & Total & $\begin{array}{l}\text { Non-CDMS } \\
\text { converter }\end{array}$ & $\mathbf{n}$ & $\begin{array}{l}\text { CDMS } \\
\text { converter }\end{array}$ & $\mathbf{n}$ & $p$ Value \\
\hline \multicolumn{7}{|l|}{ Baseline } \\
\hline Age (years), mean \pm SD & $33 \pm 10$ & $35 \pm 11$ & 50 & $31 \pm 8$ & 38 & $0.053^{\#}$ \\
\hline Sex (female), n (\%) & $60(68)$ & $29(58)$ & 50 & $31(82)$ & 38 & 0.019 * \\
\hline Monofocal syndrome, n (\%) & $84(96)$ & $48(96)$ & 50 & $36(95)$ & 38 & $0.778^{*}$ \\
\hline Optic neuritis, $n(\%)^{a}$ & $25(30)$ & $13(27)$ & 48 & $12(33)$ & 36 & $0.715^{*}$ \\
\hline Myelitis, n (\%) ${ }^{a}$ & $38(45)$ & $24(50)$ & 48 & $14(39)$ & 36 & $0.357 *$ \\
\hline Brainstem/cerebellum, n (\%) ${ }^{a}$ & $20(24)$ & $10(21)$ & 48 & $10(28)$ & 36 & $0.311^{*}$ \\
\hline Other cerebral symptom, $n(\%)^{a}$ & $1(1)$ & $1(2)$ & 48 & $0(0)$ & 36 & $0.381 *$ \\
\hline Disease duration (days) ${ }^{\mathbf{b}}$ & $12(5-34)$ & $18(7-60)$ & 50 & $7(3-20)$ & 38 & $0.003^{\S}$ \\
\hline No corticosteroid treatment before LP, $n$ (\%) & $56(69)$ & $34(77)$ & 44 & $22(59)$ & 37 & $0.329 *$ \\
\hline \multicolumn{7}{|l|}{ Brain MRI } \\
\hline Number of $\mathrm{T} 2$ hyperintense lesions & $10(3-15)$ & $8(3-15)$ & 47 & $10(7-20)$ & 35 & $0.040^{\S}$ \\
\hline Number of contrast-enhancing lesions & $1(0-2)$ & $0(0-1)$ & 44 & $2(1-3)$ & 29 & $0.002^{\S}$ \\
\hline Dissemination in space, $\mathbf{n}(\%)$ & $60(73)$ & $31(66)$ & 47 & $29(83)$ & 35 & $0.088^{*}$ \\
\hline Dissemination in time, $\mathbf{n}(\%)$ & $41(56)$ & $19(43)$ & 44 & $22(76)$ & 29 & $0.006 *$ \\
\hline Field strength (1.5 t), n (\%) & $71(87)$ & $39(83)$ & 47 & $32(91)$ & 35 & $0.338^{*}$ \\
\hline \multicolumn{7}{|l|}{ Spinal MRI } \\
\hline Number of $\mathrm{T} 2$ hyperintense lesions & $1(0-2)$ & $1(0-2)$ & 26 & $1(1-2)$ & 17 & $0.663^{\S}$ \\
\hline Number of contrast-enhancing lesions & $1(0-1)$ & $0(0-1)$ & 26 & $1(0-1)$ & 14 & $0.418^{\S}$ \\
\hline Field strength (1.5 t), n (\%) & $38(88)$ & $22(85)$ & 26 & $16(94)$ & 17 & $0.633^{*}$ \\
\hline \multicolumn{7}{|l|}{ CSF analysis } \\
\hline RBC count $(/ \mu \mathrm{l})$ & $0(0-2)$ & $0(0-8)$ & 48 & $0(0-1)$ & 37 & $0.580^{\S}$ \\
\hline WBC count $(/ \mu \mathrm{l})$ & $5(3-12)$ & $5(3-12)$ & 48 & $6(3-12)$ & 37 & $0.635^{\S}$ \\
\hline$Q_{\text {alb }}\left(\times 10^{3}\right)$ & $4.4(3.5-6.4)$ & $5.2(3.6-6.7)$ & 50 & $4.0(3.3-5.4)$ & 38 & $0.080^{\S}$ \\
\hline Oligoclonal IgG bands, n (\%) & $79(90)$ & $43(86)$ & 50 & $36(95)$ & 38 & $0.180^{*}$ \\
\hline CSF K-FLC (mg/L) & $\begin{array}{l}1.91 \\
(0.68-4.90)\end{array}$ & $\begin{array}{l}1.75 \\
(0.67-3.78)\end{array}$ & 50 & $\begin{array}{l}2.79 \\
(0.70-6.53)\end{array}$ & 38 & $0.106^{\S}$ \\
\hline Serum K-FLC (mg/L) & $\begin{array}{l}11.60 \\
(9.42-15.50)\end{array}$ & $\begin{array}{l}11.40 \\
(9.53-15.50)\end{array}$ & 50 & $\begin{array}{l}11.75 \\
(8.96-15.60)\end{array}$ & 38 & $0.886^{\S}$ \\
\hline CSF $\lambda$-FLC (mg/L) & $\begin{array}{l}0.68 \\
(0.20-2.58)\end{array}$ & $\begin{array}{l}0.67 \\
(0.20-3.74)\end{array}$ & 50 & $\begin{array}{l}0.71 \\
(0.20-2.16)\end{array}$ & 38 & $0.636^{\S}$ \\
\hline Serum $\lambda$-FLC (mg/L) & $\begin{array}{l}12.54 \\
(9.76-17.17)\end{array}$ & $\begin{array}{l}12.35 \\
(9.69-16.00)\end{array}$ & 50 & $\begin{array}{l}13.29 \\
(9.82-18.60)\end{array}$ & 38 & $0.424^{\S}$ \\
\hline Fulfillment of McDonald criteria 2017 at baseline, $n$ (\%) & $63(77)$ & $33(70)$ & 47 & $30(86)$ & 35 & $0.100 *$ \\
\hline \multicolumn{7}{|l|}{ Follow-up } \\
\hline Follow-up duration (months) & $\begin{array}{l}47 \\
(38-48)\end{array}$ & $\begin{array}{l}43.6 \\
(35.5-48.0)\end{array}$ & 50 & $\begin{array}{l}47.9 \\
(46.8-48.9)\end{array}$ & 38 & $0.001^{\S}$ \\
\hline \multicolumn{7}{|l|}{ Disease-modifying treatment } \\
\hline $\begin{array}{l}\text { DMT start before the second attack in CDMS converter or until the end of FU in } \\
\text { non-CDMS converter, } n(\%)^{c}\end{array}$ & $20(23)$ & $11(22)$ & 50 & $9(24)$ & 38 & $0.852^{*}$ \\
\hline Time to DMT start (months) & $7.0(3.7-8.4)$ & $7.4(3.0-11.2)$ & 11 & $6.9(3.8-7.9)$ & 9 & $0.790^{\S}$ \\
\hline
\end{tabular}


Table 1 Demographic, Clinical, MRI, and CSF Characteristics (continued)

\begin{tabular}{|c|c|c|c|c|c|c|}
\hline & Total & $\begin{array}{l}\text { Non-CDMS } \\
\text { converter }\end{array}$ & $\mathbf{n}$ & $\begin{array}{l}\text { CDMS } \\
\text { converter }\end{array}$ & $\mathbf{n}$ & $p$ Value \\
\hline Duration of DMT before the second attack (months) & & & & $\begin{array}{l}9.0 \\
(5.5-12.6)\end{array}$ & 9 & \\
\hline \multicolumn{7}{|l|}{ Clinical attacks } \\
\hline Time to second attack (months) ${ }^{d}$ & & & & $\begin{array}{l}11.1 \\
(4.3-22.6)\end{array}$ & 38 & \\
\hline Time to second attack in treated patients (months) ${ }^{d}$ & & & & $\begin{array}{l}18.0 \\
(12.6-24.7)\end{array}$ & 9 & \\
\hline Time to second attack in nontreated patients (months) ${ }^{d}$ & & & & $\begin{array}{l}9.8 \\
(3.1-19.4)\end{array}$ & 29 & \\
\hline Number of attacks until the end of FU & $0(0-2)$ & $0(0-0)$ & & $2(1-3)$ & 38 & $<0.001^{\S}$ \\
\hline \multicolumn{7}{|l|}{ Disability } \\
\hline EDSS score $\geq 3.0$ at yr $1, n$ (\%) & $3(4)$ & $2(5)$ & 44 & $1(3)$ & 33 & $0.734^{*}$ \\
\hline EDSS score $\geq 3.0$ at yr $2, \mathrm{n}(\%)$ & $7(10)$ & $2(6)$ & 36 & $5(15)$ & 34 & $0.202 *$ \\
\hline EDSS score $\geq 3.0$ at yr $3 / 4$ (LCF), $n$ (\%) & $8(9)$ & $2(4)$ & 50 & $6(17)$ & 36 & $0.046^{*}$ \\
\hline \multicolumn{7}{|c|}{ 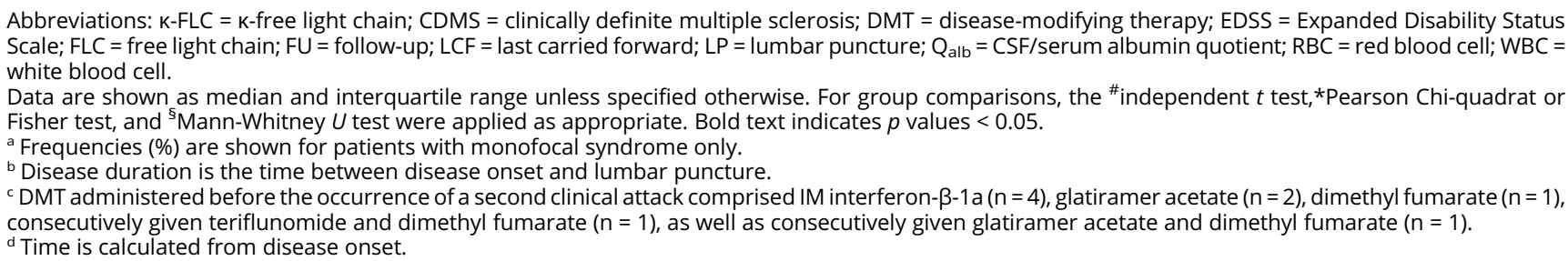 } \\
\hline
\end{tabular}

to the manufacturer's instructions. $\kappa$ - and $\lambda$-FLC concentrations are detected by latex-conjugated monoclonal antibodies to epitopes that are exposed when $\kappa$ - and $\lambda$-FLC circulate freely. ${ }^{18,19}$ All samples were measured under blinded conditions centrally at the FH Campus Wien, University of Applied Sciences.

Intrathecal synthesis of $\kappa$ - and $\lambda$-FLC was determined as previously published ${ }^{8,9}$ by the following formulas considering serum FLC concentrations and blood-CSF barrier function.

$$
\begin{aligned}
& \kappa-F L C \text { index }=\frac{\kappa-\mathrm{FLC}_{\mathrm{CSF}} / \kappa-\mathrm{FLC}_{\text {Serum }}}{\text { Albumin }_{\mathrm{CSF}} / \text { Albumin }_{\text {Serum }}} \\
& \lambda-\mathrm{FLC} \mathrm{index}=\frac{\lambda-\mathrm{FLC}_{\mathrm{CSF}} / \lambda-\mathrm{FLC}_{\text {Serum }}}{\text { Albumin }_{\mathrm{CSF}} / \text { Albumin }_{\text {Serum }}}
\end{aligned}
$$

A $\kappa$-free light chain ( $\kappa$-FLC) index $\geq 6.6$ and $\lambda$-FLC index $\geq 6.9$ denoted the presence of an intrathecal $\kappa$-FLC and $\lambda$-FLC synthesis, respectively (termed as "positive"). Index values below these cutoffs denoted the absence of an intrathecal synthesis (termed as "negative"). ${ }^{9}$

\section{Primary Research Question}

Does $\kappa$-FLC index in patients with early MS predict the time to second clinical attack? This study provides Class II evidence that in patients with early MS, high $\kappa$-FLC index is an independent risk factor for early second clinical attack.

\section{Statistical Analysis}

Statistical analysis was performed using $\mathrm{R}$ software. ${ }^{20}$ Distribution of data was assessed by the Kolmogorov-Smirnov test, and data were displayed as mean \pm SD or as median and interquartile range. For group comparisons, the Mann-Whitney $U$ test, $\chi^{2}$ test, or Fisher test was applied, as appropriate. Spearman correlation coefficient was used for correlation analysis.

To identify predictors of the time to second clinical attack, Cox regression was used including the independent variables that statistically significantly differed between patients who converted to CDMS and patients who remained stable during follow-up (nonconverters), i.e., sex, disease duration, follow-up duration, T2L, CEL (table 1), and each variable of interest, i.e., $\kappa$-FLC index, $\lambda$-FLC index, or OCB status. $p$ Values $<0.05$ were considered statistically significant. Age did not reach statistical significance at this level $(p=0.053)$, but due to findings of previous studies age was nevertheless included into Cox regression. ${ }^{7}$ In addition, corticosteroid treatment administered before lumbar puncture was considered as main effect, and as interaction effect, because an impact of corticosteroid treatment on FLC index (and OCB status) cannot be definitely excluded. Also, start of DMT was considered as main effect to control for a potential impact on time to second clinical attack.

To assess the additional performance of the $\kappa$-FLC index compared with OCB, the $\kappa-F L C$ index was added to the Cox 


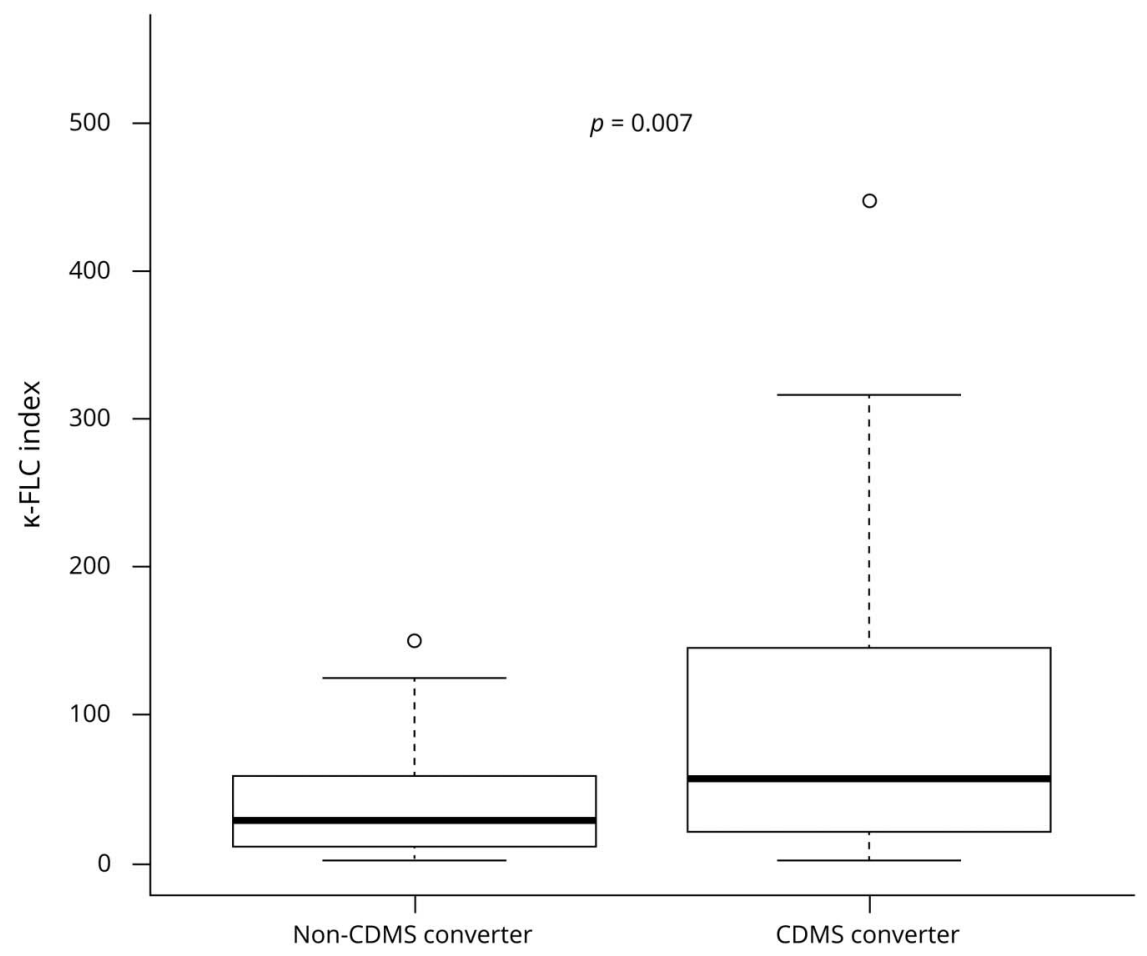

$\mathrm{k}-\mathrm{FLC}=\mathrm{k}$-free light chain; CDMS = clinically definite multiple sclerosis. regression with OCB, and the reduction of the log likelihood was tested for statistical significance. The procedure was applied vice versa too. The assumptions of the Cox regression were checked, and 2 influential observations were eliminated.

To visualize the effect of the $\kappa$-FLC index, we computed the estimated Cox regression survival probabilities separately for high and low $\kappa$-FLC index values. We calculated the 4th quintile of all $\kappa$-FLC index values (102.5). All $\kappa$-FLC index values $>100$ were thus considered as high. Low $\kappa$-FLC index values were set $\leq 100$. The average of these high and low $\kappa$-FLC index values, respectively, was used to plug into the Cox regression to compute the graph. In addition, corticosteroid treatment and DMT were set to "no treatment", sex to "female", and all other parameters were set to their median values.

An a priori computed power analysis for the Cox regression with a significance level of $5 \%$, a power of $80 \%$, a total of 9 independent variables (plus an interaction effect), and a medium effect size of the parameter of interest (according to previous findings in the literature, ${ }^{21-23}$ a Cohen $\mathrm{f}^{2}$ of 0.15 was used $^{24}$ ) revealed a necessary sample size of 55 patients to test just the parameter of interest and a sample size up to 118 for testing all parameters in the Cox regression given a corresponding medium effect size.

\section{Ethics}

The study was approved by the ethics committee of the Medical University of Innsbruck (approval number 1244/
2019). Written informed consent was obtained from all patients.

\section{Data Availability}

Anonymized data will be shared on reasonable request from any qualified investigator.

\section{Results}

A total of 88 patients at a mean age of $33 \pm 10$ years with a female predominance of $68 \%$ were included into the study. Most patients showed a monofocal syndrome with myelitis (45\%), followed by optic neuritis (30\%), affection of brainstem/ cerebellum $(24 \%)$, or other topography (1\%). OCBs were positive in 79 (90\%) of 88 patients. During follow-up of median 47 months, 38 (43\%) of 88 patients converted to CDMS. Twenty (23\%) of 88 patients received early DMT, between disease onset and the date of conversion to CDMS for converters, or during follow-up for nonconverters. Of 86 patients with available EDSS data at follow-up, 8 (9\%) reached an EDSS score of 3.0. Detailed demographic and clinical characteristics and CSF and MRI findings are displayed in table 1.

\section{K-FLC Index Is Increased in Patients Who Convert to Clinically Definite Multiple Sclerosis} $\kappa$-FLC index determined at baseline had a median of 36.5 and was denoted positive in $76(86 \%)$ of 88 patients. $\kappa$-FLC index significantly correlated with WBC count $(r=0.55, p<0.001)$ and the number of T2Ls $(r=0.26, p=0.020)$ and CELs $(r=$ 
Table 2 k-FLC Index Depending on OCB Status and Conversion to CDMS

\begin{tabular}{llllll}
\hline & \multicolumn{2}{l}{ Non-CDMS converter } & & \multicolumn{2}{l}{ CDMS converter } \\
\cline { 2 - 3 } & OCB negative & OCB positive & & OCB negative & OCB positive \\
\hline $\mathbf{n}$ & 7 & 43 & 2 & 36 \\
\hline $\mathbf{k}-\mathrm{FLC}$ index & $5.3(2.7-6.1)$ & $39.0(19.4-66.9)$ & $4.6(1.7-7.5)$ & $67.7(29.2-146.3)$ \\
\hline
\end{tabular}

Abbreviations: $\mathrm{CDMS}=$ clinically definite multiple sclerosis; $\mathrm{OCB}=$ oligoclonal bands; $\mathrm{k}-\mathrm{FLC}=\mathrm{k}$-free light chain .

$\mathrm{K}$-FLC index was significantly higher in OCB-positive patients who converted to CDMS during follow-up compared with OCB-positive nonconverters $(p=0.020)$.

Data are shown as median and interquartile range.

$0.25, p<0.032)$ and was higher in patients with CSF-restricted OCB compared with those without OCB $(p<0.001)$. $\kappa$-FLC index was significantly elevated in patients who converted to CDMS during follow-up compared with nonconverters (figure 1 ), but did not differ between patients who reached an EDSS score of 3 and those who remained below. $\kappa$-FLC index was higher in the group of OCB-positive CDMS converters compared with OCB-positive nonconverters (table 2).

$\lambda$-FLC index at baseline had a median of 12.9 and was denoted positive in $55(63 \%)$ of 88 patients. $\lambda$-FLC index did not show any statistically significant correlations with WBC count and number of T2Ls and CELs, but was elevated in OCB-positive patients compared with OCB-negative patients $(p=0.001)$. $\lambda$-FLC index did not differ between patients converting to CDMS during follow-up and nonconverters (figure e-1, links. lww.com/NXI/A481) and also did not differ with respect to progression to an EDSS score of 3.

\section{High k-FLC Index Is Associated With Shorter Time to Clinically Definite Multiple Sclerosis}

To investigate whether $\kappa$-FLC index predicts the time to CDMS conversion, multivariate Cox regression model including age, sex, number of T2Ls, number of CELs, disease duration and follow-up duration, and the administration of corticosteroids before lumbar puncture and of DMT during follow-up was performed. $\kappa$-FLC index was an independent risk factor for the time to CDMS conversion with a hazard ratio (HR) of 1.13 for an increase of $\kappa$-FLC index by 10 ( $p=$ 0.009 ), i.e., an increase of $\kappa$-FLC index by 10 means a $13 \%$ higher risk for conversion to CDMS (table 3). Patients with a $\kappa$-FLC index $>100$ (median value 147) at baseline had a twice as high probability for a second clinical attack within 12 months than patients with a low $\mathrm{k}$-FLC index (median value 28); within 24 months, the estimated chance for a second clinical attack in patients with high $\kappa$-FLC index at baseline was 4 times as high compared with patients with low $\kappa$-FLC index. The median time to second attack was 11 months in patients with high $\kappa$-FLC index whereas 36 months in those with low $\kappa$-FLC index (figure 2). The distribution of $\kappa$-FLC index values and the absolute number of patients within the high and low $\kappa$-FLC index categories is shown in figure e-2, links.lww.com/NXI/A481.

$\lambda$-FLC index was not a statistically significant predictor of the time to CDMS conversion in the multivariate Cox regression

Table 3 Regression Analysis Identifiying k-FLC Index as Predictor for Time to Second Clinical Attack

\begin{tabular}{|c|c|c|c|c|c|}
\hline & Coefficient & Hazard ratio & Standard error & $z$ & $p$ Value \\
\hline Age (years) & -0.0269 & 0.9734 & 0.0271 & -0.992 & 0.321 \\
\hline Sex (ref: male) & -0.2160 & 0.8057 & 0.5478 & -0.394 & 0.693 \\
\hline Disease duration (days) & -0.0215 & 0.9787 & 0.0123 & -1.756 & 0.079 \\
\hline Follow-up duration (months) & 0.1523 & 1.1645 & 0.0619 & 2.461 & 0.014 \\
\hline Number of T2 hyperintense MRI lesions & -0.0012 & 0.9988 & 0.0143 & -0.087 & 0.930 \\
\hline Number of T1 contrast-enhancing MRI lesions & 0.1198 & 1.1273 & 0.1329 & 0.902 & 0.367 \\
\hline DMT administration & -0.3952 & 0.6736 & 0.6837 & -0.578 & 0.563 \\
\hline Corticosteroid treatment prior LP & 0.7119 & 2.0379 & 0.6585 & 1.081 & 0.280 \\
\hline K-FLC index & 0.0125 & 1.0126 & 0.0048 & 2.600 & 0.009 \\
\hline K-FLC index: corticosteroid treatment prior LP & -0.0026 & 0.9974 & 0.0086 & -0.301 & 0.764 \\
\hline
\end{tabular}

Abbreviations: $\mathrm{k}-\mathrm{FLC}=\mathrm{k}$-free light chain; DMT = disease-modifying therapy; $\mathrm{LP}=$ lumbar puncture.

Disease duration was the time between symptom onset and lumbar puncture. Age was determined at the time of lumbar puncture. The number of MRI lesions was also determined at baseline. Follow-up duration was the time between disease onset and the last clinical visit. DMT administration was determined until occurrence of the second clinical attack or end of follow-up, respectively. Bold text indicates $p$ values $<0.05$. 


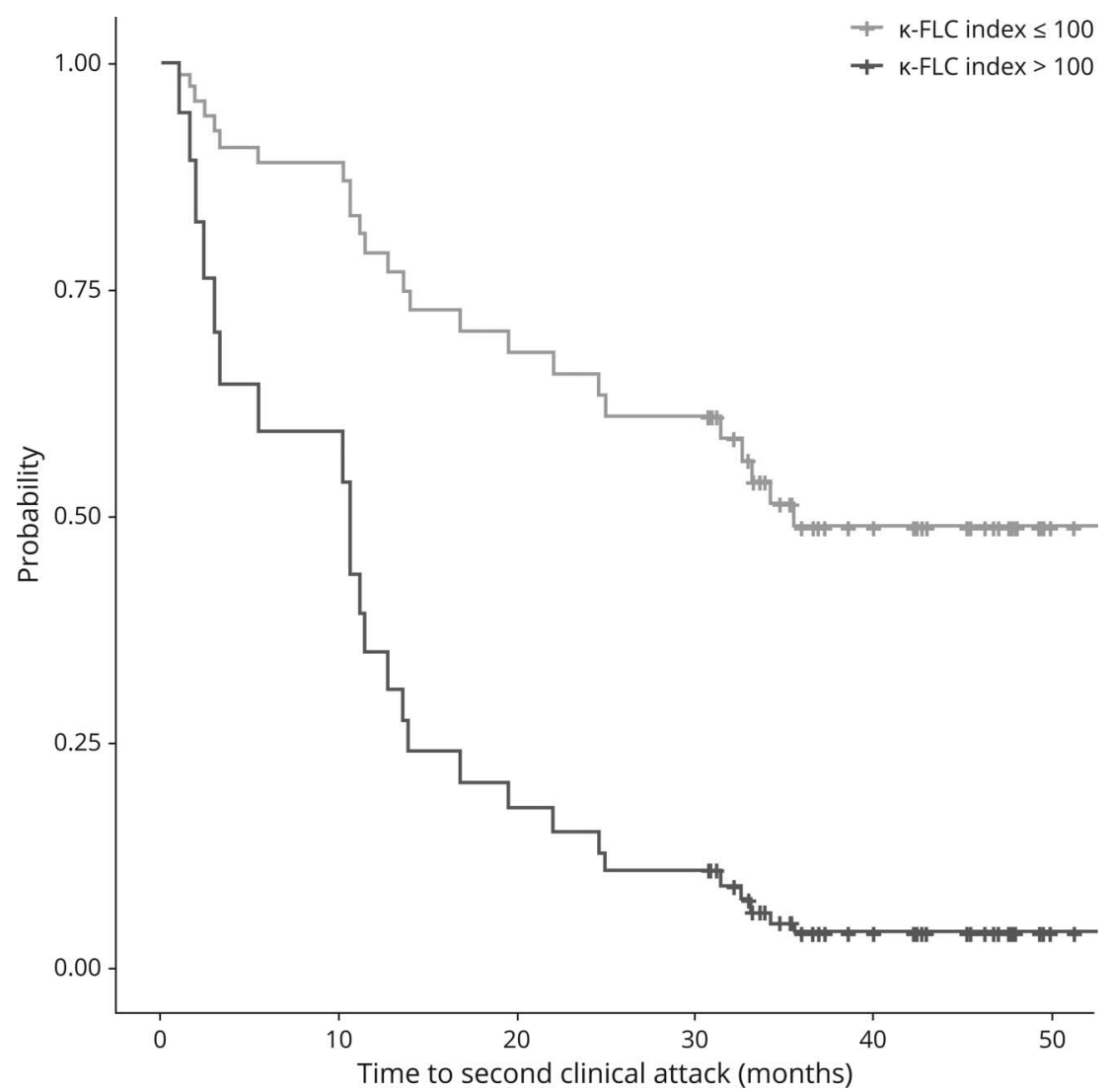

The probability of development of a second clinical attack, i.e., conversion to CDMS, during the 4 -year follow-up period was higher in the high $\mathrm{k}$-FLC index $(>100)$ group $(n=19)$ than in the low $k-F L C$ index $(\leq 100)$ group $(\mathrm{n}=69 ; p=0.009) . \mathrm{k}$-FLC $=\mathrm{k}$-free light chain; CDMS = clinically definite multiple sclerosis. model (table e-1, links.lww.com/NXI/A484). Further analyses showed a prognostic value of the $\kappa / \lambda$ ratio (table e- 2 , links. lww.com/NXI/A484), but not for OCB status (table e-3, links.lww.com/NXI/A484).

Adding of $\kappa$-FLC index to the Cox regression already including OCB improved the model for the time to second clinical attack ( $p=0.008$; table e-3, links.lww.com/NXI/A484), whereas OCB status did not in the presence of $\kappa$-FLC index $(p=0.621)$.

Various sensitivity analyses support the robustness of our findings. First, a Cox regression performed only in the subgroup of OCB-positive patients still revealed a statistically significant effect of $\kappa$-FLC index for predicting the time to second clinical attack (table e-4, links.lww.com/NXI/A484). Further analyses, including only the subgroup of patients without DMT (table e-5, links.lww.com/NXI/A484), or considering also the type of disease manifestation as additional independent control variable (table e-6, links.lww.com/ NXI/A484), consistently showed an effect of $\kappa$-FLC index.

\section{Discussion}

In this study, we demonstrated that $\kappa$-FLC index measured at the time of diagnostic lumbar puncture in patients with a first demyelinating event of the CNS predicts the time to a second clinical attack, that is conversion to CDMS, independent of other prognostic factors including load and activity of brain MRI lesions and independent of administered DMT. Furthermore, we showed that $\kappa$-FLC index has a prognostic value in contrast to determination of CSF-restricted OCB.

Free light chains that occur either as $\kappa$ - or $\lambda$-isotype are secreted in excess over intact immunoglobulins by terminally differentiated $\mathrm{B}$ cells ${ }^{25}$ and accumulate in the CSF in case of intrathecal inflammation, as this is the case in chronic inflammatory CNS diseases such as MS. Intrathecal $\kappa$-FLC synthesis determined by an increased $\kappa$-FLC index was proven as a reliable diagnostic biomarker in MS performing similar to OCB. ${ }^{8,9,26-30}$ However, only a few previous studies have investigated the prognostic value of $\kappa$-FLC in MS, some with considerable limitations. These include measuring $\kappa$-FLC concentrations only in $\mathrm{CSF}^{10,31-33}$ or calculating the CSF/serum ratio ${ }^{10,34}$ without reference to the blood-CSF barrier function and mixing different MS disease courses within 1 group for statistical analysis. ${ }^{11,32,35}$ Furthermore, studies investigating the effect of $\kappa$-FLC index independent of already known prognostic factors including demographics, clinical variables, and MRI findings are still lacking. ${ }^{36}$

Here, we showed in a multivariate analysis that in patients after the first demyelinating event, the $\kappa$-FLC index allows risk 
stratification. This multivariate approach is critical to identify the independent prognostic effect of $\kappa$-FLC index and to weigh its impact on the outcome in comparison with the remaining baseline characteristics. Our cohort, which is representative for patients with a first demyelinating event, shows that an increase of $\kappa$-FLC index by 10 indicates an increased risk by approximately $13 \%$ for a second clinical attack; and the probability for a second clinical attack within 12 months in patients with high $\kappa$-FLC index $(>100)$ is twice as high as in patients with low $\kappa$-FLC index.

Although OCBs also imply some independent prognostic value predicting time to a second clinical attack, ${ }^{7,21}$ the determination of $\kappa$-FLC index has considerable advantages. First, $\kappa$-FLCs are measured by nephelometry, which is-in contrast to the detection of $\mathrm{OCB}$ - an easy, reliable, labor-saving, and costefficient method. ${ }^{19}$ Second, $\kappa$-FLC index returns a metric result covering a range from approximately 1 up to $500,{ }^{8}$ i.e., it is a quantitative variable, whereas OCB status is dichotomous returning either a positive or negative result as assessed by visual inspection. ${ }^{17}$ OCBs were detected in $95 \%$ of CDMS converters, whereas nonconverters were OCB positive also in $86 \%$ of cases. As a continuous variable, $\kappa$-FLC index overcomes the weak performance of OCB by further stratification. $\kappa$-FLC index had a median of 68 in OCB-positive CDMS converters, whereas the median was 39 in OCB-positive nonconverters (table 2). Testing for log-likelihood reduction by adding either $\kappa$-FLC index or OCB to the Cox regression including the corresponding other parameter already confirmed the superior predictive value of $\kappa$-FLC index. Also, when Cox regression analysis was performed in the subgroup of OCB-positive patients only, $\kappa$-FLC index was still a statistically significant predictor with statistically the same effect size. Although other studies reported an association with clinical outcome by counting the number of CSF-restricted $\mathrm{OCB},{ }^{37,38}$ isoelectric focusing is a qualitative method and $\mathrm{OCB}$ counting showed limited reliability due to interrater variability. ${ }^{39}$ The above discussed aspects and differences between FLC and OCB determination should be considered, especially when a wide use in clinical practice is aspired and when results are compared across laboratories.

Of interest, $\kappa$-FLC index differed between CDMS converters and nonconverters, whereas the absolute CSF $\kappa$-FLC concentrations were similar in these 2 groups. This finding highlights again the importance of determining intrathecal -FLC synthesis, e.g., by calculating the $\kappa$-FLC index. Some authors have suggested using absolute CSF FLC concentrations, ${ }^{40}$ as the contribution of blood-derived FLC to the total CSF FLC concentration is low in cases with intrathecal synthesis. In fact, the intrathecal fraction of $\mathrm{k}$-FLC is greater than $80 \%$ in most patients with MS. ${ }^{8}$ However, there are some confounders such as grossly elevated serum FLC levels in some cases leading to relatively low intrathecal FLC proportions or a higher degree of blood-CSF barrier dysfunction. In line with this, we could recently show that the diagnostic value of FLC index is superior to that of absolute CSF FLC concentrations. $^{41}$
There are some limitations to this study. First, CSF and serum samples have been stored for a median of 6 years at $-80^{\circ} \mathrm{C}$ before analysis. However, the effect of freezing on FLC concentrations $\left(\right.$ at $-20^{\circ} \mathrm{C}$ ) has been reported to be irrelevant. ${ }^{42}$ Furthermore, in the present study, we used $\kappa$-FLC index that includes the ratio of CSF and serum $\kappa$-FLC concentrations for its calculation and, thus, is less prone to variations. Also, adding storage time to the multivariate Cox regression model did not change the overall result. Not all CSF and serum samples were collected before administration of corticosteroids. It has been recently shown that high-dose corticosteroids resulted in lower serum FLC concentrations, however, CSF levels and more importantly the FLC index were not affected. ${ }^{43}$ In the present study, the proportion of patients with corticosteroid treatment before lumbar puncture did not differ between patients who converted to CDMS and nonconverters. Considering corticosteroid treatment as independent variable as well as its interaction effect with $\kappa$-FLC index in the Cox regression analysis confirms that corticosteroid treatment does neither impact on the time to CDMS conversion nor on $\kappa$-FLC index. The $\kappa$-FLC index estimates were comparable between the groups (figure e-3, links.lww.com/NXI/ A481). Nevertheless, it has to be stated that an effect of corticosteroids cannot be ultimately excluded due to the limited number of patients. The majority of patients did not receive DMT during follow-up, and the proportion of patients with early DMT as well as the time to DMT initiation did not significantly differ between CDMS converters and non-CDMS converters. Anyway, we considered DMT as an independent variable in the Cox regression analysis, performed subgroup analysis including only patients without DMT, and consistently observed no DMT effect on the time to second clinical attack. An interaction effect between DMT and $\kappa$-FLC index was not considered, as all patients started DMT after lumbar puncture.

In clinical practice, the benefit of a reliable biomarker-either singular or in combination with others-is that patients with early MS with a higher risk for further disease activity, that is, e.g., shorter time to a second attack, could be advised to start DMT early or use highly effective DMT. There is evidence that the time to the second attack has a prognostic impact on long-term disability ${ }^{44,45}$ and that early treatment significantly delays conversion to CDMS as well as disability progression. ${ }^{46-48}$ Conversely, there is a certain proportion of patients who show a mild disease course and who may not need a potentially harmful, psychologically distressing, and, last but not least, costly DMT. Current evidence suggests that $\kappa$-FLC index is a reliable prognostic biomarker that might replace OCB determination taking us one step closer to tailored medicine in MS. Further studies in a multicenter setting including a higher number of patients are required to replicate the independent prognostic value of $\kappa$-FLC index in early MS. Also, besides clinical end points such as time to second clinical attack, it might be of interest to investigate how $\kappa$-FLC index performs for predicting MRI activity in the early MS disease course.

\section{Study Funding}

No targeted funding reported. 


\section{Disclosure}

$\mathrm{K}$. Berek has participated in meetings sponsored by and received travel funding from Roche. G. Bsteh has participated in meetings sponsored by, received speaker honoraria or travel funding from Biogen, Celgene, Lilly, Merck, Novartis, Sanofi-Genzyme, and Teva, and received honoraria for consulting Biogen, Celgene, Merck, Novartis, Roche, and Teva. M. Auer received speaker honoraria and/or travel grants from Biogen, Novartis, Merck, and Sanofi. F. Di Pauli has participated in meetings sponsored by and received honoraria (lectures, advisory boards, and consultations) or travel funding from Bayer, Biogen, Celgene, Merck, Novartis, Sanofi-Genzyme, Roche, and Teva. A. Grams has nothing to disclose. D. Milosavljevic has participated in meetings sponsored by Siemens. P. Poskaite has nothing to disclose. C. Schnabl has participated in meetings sponsored by Siemens. S. Wurth has participated in meetings sponsored by and received honoraria or travel funding from Allergan, Biogen, Ipsen Pharma, Merck, Novartis, Roche, Sanofi-Genzyme, and Teva. A. Zinganell has participated in meetings sponsored by and received speaking honoraria or travel funding from Biogen, Merck, Sanofi-Genzyme, and Teva.T. Berger has participated in the last 2 years in meetings sponsored by and received honoraria (lectures, advisory boards, and consultations) from pharmaceutical companies marketing treatments for multiple sclerosis: Almirall, Biogen, Bionorica, Celgene, MedDay, Merck, Novartis, Roche, Sanofi Aventis/ Genzyme, TG Therapeutics, and Teva. His institution has received financial support in the last 2 years by unrestricted research grants (Biogen, Novartis, Sanofi-Aventis/Genzyme, Roche, and Teva) and for participation in clinical trials in multiple sclerosis sponsored by Alexion, Bayer, Biogen, Merck, Novartis, Roche, Sanofi-Aventis/Genzyme, and Teva. J. Walde has nothing to disclose. F. Deisenhammer has participated in meetings sponsored by or received honoraria for acting as an advisor/speaker for Alexion, Almirall, Biogen, Celgene, Genzyme-Sanofi, Merck, Novartis Pharma, Roche, and Teva. His institution has received research grants from Biogen and Genzyme-Sanofi. He is section editor of the MSARD Journal (Multiple Sclerosis and Related Disorders). H. Hegen has participated in meetings sponsored by, received speaker honoraria or travel funding from Bayer, Biogen, Merck, Novartis, Sanofi-Genzyme, Siemens, Teva, and received honoraria for acting as consultant for Biogen and Teva. Go to Neurology.org/NN for full disclosures.

\section{Publication History}

Received by Neurology: Neuroimmunology \& Neuroinflammation November 6, 2020. Accepted in final form March 2, 2021.

Appendix Authors

\begin{tabular}{|c|c|c|}
\hline Authors & Location & Contribution \\
\hline Klaus Berek, MD & $\begin{array}{l}\text { Department of Neurology, } \\
\text { Medical University of } \\
\text { Innsbruck, Austria }\end{array}$ & $\begin{array}{l}\text { Drafting/revision of the } \\
\text { manuscript for content, } \\
\text { including medical writing } \\
\text { for content, and major role } \\
\text { in the acquisition of data }\end{array}$ \\
\hline
\end{tabular}

Appendix (continued)

\begin{tabular}{lll}
\hline Authors & Location & Contribution \\
\hline $\begin{array}{l}\text { Gabriel Bsteh, } \\
\text { MD, PhD }\end{array}$ & $\begin{array}{l}\text { Department of Neurology, } \\
\text { Medical University of } \\
\text { Innsbruck, Austria }\end{array}$ & $\begin{array}{l}\text { Drafting/revision of the } \\
\text { manuscript for content, } \\
\text { including medical writing } \\
\text { for content, and major role } \\
\text { in the acquisition of data }\end{array}$ \\
\hline $\begin{array}{l}\text { Michael Auer, } \\
\text { MD, PhD }\end{array}$ & $\begin{array}{l}\text { Department of Neurology, } \\
\text { Medical University of } \\
\text { Innsbruck, Austria }\end{array}$ & $\begin{array}{l}\text { Drafting/revision of the } \\
\text { manuscript for content, } \\
\text { including medical writing } \\
\text { for content }\end{array}$ \\
\hline
\end{tabular}

Franziska Di

Pauli, MD, PhD

Department of Neurology, Drafting/revision of the Medical University of manuscript for content, Innsbruck, Austria including medical writing for content

\begin{tabular}{lll}
\hline Astrid Grams, & $\begin{array}{l}\text { Department of Neurology, } \\
\text { Medical University of } \\
\text { Innsbruck, Austria }\end{array}$ & $\begin{array}{l}\text { Drafting/revision of the } \\
\text { manuscript for content, } \\
\text { including medical writing } \\
\text { for content, and major role } \\
\text { in the acquisition of data }\end{array}$ \\
& &
\end{tabular}

\begin{tabular}{lll}
\hline Dejan & FH Campus Wien, & Drafting/revision of the \\
Milosavljevic, & University of Applied & manuscript for content, \\
BSc, MSc & Sciences, Vienna, Austria & $\begin{array}{l}\text { including medical writing } \\
\text { for content, and major } \\
\text { role in the acquisition of } \\
\text { data }\end{array}$ \\
& & \\
&
\end{tabular}

\begin{tabular}{lll}
\hline Paulina & Department of & $\begin{array}{l}\text { Drafting/revision of the } \\
\text { Poskaite, MD }\end{array}$ \\
$\begin{array}{ll}\text { Neuroradiology, Medical } \\
\text { University of Innsbruck, } \\
\text { including medical writing } \\
\text { for content, and major } \\
\text { role in the acquisition of } \\
\text { data }\end{array}$ \\
\hline
\end{tabular}

\begin{tabular}{lll}
\hline $\begin{array}{l}\text { Christine } \\
\text { Schnabl, MSc }\end{array}$ & $\begin{array}{l}\text { FH Campus Wien, } \\
\text { University of Applied } \\
\text { Sciences, Vienna, Austria }\end{array}$ & $\begin{array}{l}\text { Drafting/revision of the } \\
\text { manuscript for content, } \\
\text { including medical writing } \\
\text { for content }\end{array}$
\end{tabular}

Sebastian

Wurth, MD Medical University of Graz, manuscript for content, Austria including medical writing for content

\begin{tabular}{lll}
\hline Anne Zinganell, & Department of Neurology, & Drafting/revision of the \\
MD & Medical University of & manuscript for content, \\
Innsbruck, Austria & $\begin{array}{l}\text { including medical writing } \\
\text { for content }\end{array}$
\end{tabular}

Thomas Berger, Department of Neurology, Drafting/revision of the MD, MSc Medical University of manuscript for content, Vienna, Austria including medical writing for content

\begin{tabular}{|c|c|c|}
\hline $\begin{array}{l}\text { Janette Walde, } \\
\text { PhD }\end{array}$ & $\begin{array}{l}\text { Department of Statistics, } \\
\text { Faculty of Economics and } \\
\text { Statistics, University of } \\
\text { Innsbruck, Austria }\end{array}$ & $\begin{array}{l}\text { Drafting/revision of the } \\
\text { manuscript for content, } \\
\text { including medical writing } \\
\text { for content, and analysis or } \\
\text { interpretation of data }\end{array}$ \\
\hline
\end{tabular}

\begin{tabular}{lll}
\hline Florian & Department of Neurology, & Drafting/revision of the \\
Deisenhammer, & Medical University of & manuscript for content, \\
MD, MSc & Innsbruck, Austria & $\begin{array}{l}\text { including medical writing } \\
\text { for content }\end{array}$ \\
& &
\end{tabular}

Harald Hegen, Department of Neurology, Drafting/revision of the PD, MD, PhD Medical University of manuscript for content, Innsbruck, Austria including medical writing for content; major role in the acquisition of data; study concept or design; and analysis or interpretation of data 


\section{References}

1. Compston A, Coles A. Multiple sclerosis. Lancet. 2002;359(9313):1221-1231.

2. Hauser SL, Cree BAC. Treatment of multiple sclerosis: a review. Am J Med. 2020; 133(12):1380-1390.e2.

3. Giovannoni G. Disease-modifying treatments for early and advanced multiple sclerosis: a new treatment paradigm. Curr Opin Neurol. 2018;31(3):233-243.

4. Weinshenker BG, BASS B, Rice GP, et al. The natural history of multiple sclerosis: a geographically based study. I. Clinical course and disability. Brain. 1989;112(pt 1):133-146

5. Ontaneda D, Tallantyre E, Kalincik T, Planchon SM, Evangelou N. Early highly effective versus escalation treatment approaches in relapsing multiple sclerosis. Lancet Neurol. 2019;18(10):973-980.

6. Bsteh G, Hegen H, Dosser C, et al. To treat or not to treat: sequential individualized treatment evaluation in relapsing multiple sclerosis. Mult Scler Relat Disord. 2019;39:101908.

7. Tintore M, Rovira A, Río J, et al. Defining high, medium and low impact prognostic factors for developing multiple sclerosis. Brain. 2015;138(Pt 7):1863-1874.

8. Presslauer S, Milosavljevic D, Huebl W, et al. Validation of kappa free light chains as a diagnostic biomarker in multiple sclerosis and clinically isolated syndrome: a multicenter study. Mult Scler. 2016;22(4):502-510.

9. Leurs CE, Twaalfhoven $\mathrm{H}$, Lissenberg-Witte BI, et al. Kappa free light chains is a valid tool in the diagnostics of MS: a large multicenter study. Mult Scler. 2020;26(8):912-923.

10. Makshakov G, Nazarov V, Kochetova O, Surkova E, Lapin S, Evdoshenko E. Diagnostic and prognostic value of the cerebrospinal fluid concentration of immunoglobulin free light chains in clinically isolated syndrome with conversion to multiple sclerosis. PLoS One. 2015;10(11):e0143375.

11. Gaetani L, Di Carlo M, Brachelente G, et al. Cerebrospinal fluid free light chains compared to oligoclonal bands as biomarkers in multiple sclerosis. J Neuroimmunology. 2020;339:577108.

12. Thompson AJ, Banwell BL, Barkhof F, et al. Diagnosis of multiple sclerosis: 2017 revisions of the McDonald criteria. Lancet Neurol. 2018;17(2):162-173.

13. Kurtzke JF. Rating neurologic impairment in multiple sclerosis: an expanded disability status scale (EDSS). Neurology. 1983;33(11):1444-1452.

14. Deisenhammer F, Bartos A, Egg R, et al. Guidelines on routine cerebrospinal fluid analysis. Report from an EFNS task force. Eur J Neurol. 2006;13(9):913-922.

15. Auer M, Hegen H, Zeileis A, Deisenhammer F. Quantitation of intrathecal immunoglobulin synthesis-a new empirical formula. Eur J Neurol. 2016;23(4):713-721.

16. Hegen H, Zinganell A, Auer M, Deisenhammer F. The clinical significance of single or double bands in cerebrospinal fluid isoelectric focusing. A retrospective study and systematic review. PLoS One. 2019;14(4):e0215410.

17. Freedman MS, Thompson EJ, Deisenhammer F, et al. Recommended standard of cerebrospinal fluid analysis in the diagnosis of multiple sclerosis: a consensus statement. Arch Neurol. 2005;62:865-870.

18. Hoedemakers RMJ, Pruijt JFM, Hol S, et al. Clinical comparison of new monoclonal antibody-based nephelometric assays for free light chain kappa and lambda to polyclonal antibody-based assays and immunofixation electrophoresis. Clin Chem Lab Med. 2011;50(3):489-495.

19. Velthuis Te H, Knop I, Stam P, et al. N Latex FLC—new monoclonal highperformance assays for the determination of free light chain kappa and lambda. Clin Chem Lab Med. 2011;49(8):1323-1332.

20. R Core Team. R: A Language and Environment for Statistical Computing [Internet]. $\mathrm{R}$ Foundation for Statistical Computing; 2018. Available from: R-project.org.

21. Kuhle J, Disanto G, Dobson R, et al. Conversion from clinically isolated syndrome to multiple sclerosis: a large multicentre study. Mult Scler. 2015;21(8):1013-1024.

22. Tintore M, Rovira A, Rio J, et al. Do oligoclonal bands add information to MRI in first attacks of multiple sclerosis? Neurology. 2008;70(13 pt 2):1079-1083.

23. Dalla Costa G, Martinelli V, Sangalli F, et al. Prognostic value of serum neurofilaments in patients with clinically isolated syndromes. Neurology. 2019;92(7):e733-e741.

24. Cohen J. Statistical Power Analysis for the Behavioral Sciences. Lawrence Erlbaum Associates Inc; 1988.

25. Nakano T, Matsui M, Inoue I, Awata T, Katayama S, Murakoshi T. Free immunoglobulin light chain: its biology and implications in diseases. Clin Chim Acta. 2011; 412(11-12):843-849.
26. Christiansen M, Gjelstrup MC, Stilund M, Christensen T, Petersen T, Jon Møller H. Cerebrospinal fluid free kappa light chains and kappa index perform equal to oligoclonal bands in the diagnosis of multiple sclerosis. Clin Chem Lab Med. 2018;57(2): 210-220.

27. Crespi I, Vecchio D, Serino $\mathrm{R}$, et al $\mathrm{K}$ index is a reliable marker of intrathecal synthesis, and an alternative to IgG index in multiple sclerosis diagnostic work-up. J Clin Med. 2019;8(4):446.

28. Emersic A, Anadolli V, Krsnik M, Rot U. Intrathecal immunoglobulin synthesis: the potential value of an adjunct test. Clin Chim Acta. 2019;489:109-116.

29. Senel M, Mojib-Yezdani F, Braisch U, et al. CSF free light chains as a marker of intrathecal immunoglobulin synthesis in multiple sclerosis: a blood-CSF barrier related evaluation in a large cohort. Front Immunol Front. 2019;10:641.

30. Presslauer S, Milosavljevic D, Brücke T, Bayer P, Hübl W, Hübl W. Elevated levels of kappa free light chains in CSF support the diagnosis of multiple sclerosis. J Neurol. 2008;255(10):1508-1514

31. Rinker JR, Trinkaus K, Cross AH. Elevated CSF free kappa light chains correlate with disability prognosis in multiple sclerosis. Neurology. 2006;67(7):1288-1290.

32. Rudick RA, Medendorp SV, Namey M, Boyle S, Fischer J. Multiple sclerosis progression in a natural history study: predictive value of cerebrospinal fluid free kappa light chains. Mult Scler J. 1995;1(3):150-155.

33. Villar LM, Espiño M, Costa-Frossard L, Muriel A, Jiménez J, Álvarez-Cermeño JC. High levels of cerebrospinal fluid free kappa chains predict conversion to multiple sclerosis. Clin Chim Acta. 2012;413(23-24):1813-1816.

34. Senel M, Tumani H, Lauda F, et al. Cerebrospinal fluid immunoglobulin kappa light chain in clinically isolated syndrome and multiple sclerosis. PLoS One. 2014;9(4):e88680.

35. Vecchio D, Crespi I, Virgilio E, et al. Kappa free light chains could predict early disease course in multiple sclerosis. Mult Scler Relat Disord. 2019;30:81-84.

36. Salavisa M, Paixão $\mathrm{P}$, Ladeira $\mathrm{AF}$, et al. Prognostic value of kappa free light chains determination in first-ever multiple sclerosis relapse. J Neuroimmunology. 2020;347: 577355 .

37. Avasarala JR, Cross AH, Trotter JL. Oligoclonal band number as a marker for prognosis in multiple sclerosis. Arch Neurol. 2001;58(12):2044-2045.

38. Dalla Costa G, Passerini G, Messina MJ, et al. Clinical significance of the number of oligoclonal bands in patients with clinically isolated syndromes. J Neuroimmunology. 2015;289:62-67.

39. Franciotta D, Avolio C, Lolli F. Between-laboratory variability in oligoclonal IgG band numbering. Clin Chem. 2005;51(1):270-272.

40. Gurtner KM, Shosha E, Bryant SC, et al. CSF free light chain identification of demyelinating disease: comparison with oligoclonal banding and other CSF indexes. Clin Chem Lab Med. 2018;56(7):1071-1080.

41. Hegen H, Walde J, Milosavljevic D, et al. Free light chains in the cerebrospinal fluid. Comparison of different methods to determine intrathecal synthesis. Clin Chem Lab Med. 2019;57(10):1574-1586.

42. Hörber S, Klein R, Peter A. Effects of long-term storage on serum free light chain stability. Clin Lab. 2019;65(5). doi:10.7754/Clin.Lab.2018.181107.

43. Konen FF, Wurster $\mathrm{U}$, Witte $\mathrm{T}$, et al. The impact of immunomodulatory treatment on kappa free light chains as biomarker in neuroinflammation. Cells. 2020;9(4):842.

44. Scalfari A, Neuhaus A, Degenhardt A, et al. The natural history of multiple sclerosis: geographically based study 10: relapses and long-term disability. Brain. 2010;133(pt 7):1914-1929.

45. Tremlett H, Yousefi M, Devonshire V, Rieckmann P, Zhao Y. UBC Neurologists. Impact of multiple sclerosis relapses on progression diminishes with time. Neurology. 2009;73(20):1616-1623.

46. Kappos L, Polman CH, Freedman MS, et al. Treatment with interferon beta- $1 \mathrm{~b}$ delays conversion to clinically definite and McDonald MS in patients with clinically isolated syndromes. Neurology. 2006;67(7):1242-1249.

47. Comi G, Filippi M, Barkhof F, et al. Effect of early interferon treatment on conversion to definite multiple sclerosis: a randomised study. Lancet. 2001;357(9268): 1576-1582.

48. Jacobs LD, Beck RW, Simon JH, et al. Intramuscular interferon beta-1a therapy initiated during a first demyelinating event in multiple sclerosis. CHAMPS Study Group. New Engl J Med. 2000;343(13):898-904. 


\title{
Neurology \\ Neuroimmunology \& Neuroinflammation
}

\author{
Kappa-Free Light Chains in CSF Predict Early Multiple Sclerosis Disease Activity \\ Klaus Berek, Gabriel Bsteh, Michael Auer, et al. \\ Neurol Neuroimmunol Neuroinflamm 2021;8; \\ DOI 10.1212/NXI.0000000000001005
}

This information is current as of May 28, 2021

\section{Updated Information \& Services}

References

Citations

Subspecialty Collections

Permissions \& Licensing

Reprints including high resolution figures, can be found at: http://nn.neurology.org/content/8/4/e1005.full.html

This article cites 46 articles, 1 of which you can access for free at: http://nn.neurology.org/content/8/4/e1005.full.html\#\#ref-list-1

This article has been cited by 1 HighWire-hosted articles: http://nn.neurology.org/content/8/4/e1005.full.html\#\#otherarticles

This article, along with others on similar topics, appears in the following collection(s):

Cerebrospinal Fluid

http://nn.neurology.org//cgi/collection/cerebrospinal_fluid

Class II

http://nn.neurology.org//cgi/collection/class_ii

Multiple sclerosis

http://nn.neurology.org//cgi/collection/multiple_sclerosis

Information about reproducing this article in parts (figures,tables) or in its entirety can be found online at:

http://nn.neurology.org/misc/about.xhtml\#permissions

Information about ordering reprints can be found online: http://nn.neurology.org/misc/addir.xhtml\#reprintsus

Neurol Neuroimmunol Neuroinflamm is an official journal of the American Academy of Neurology.

Published since April 2014, it is an open-access, online-only, continuous publication journal. Copyright

Copyright (C) 2021 The Author(s). Published by Wolters Kluwer Health, Inc. on behalf of the American

Academy of Neurology.. All rights reserved. Online ISSN: 2332-7812.

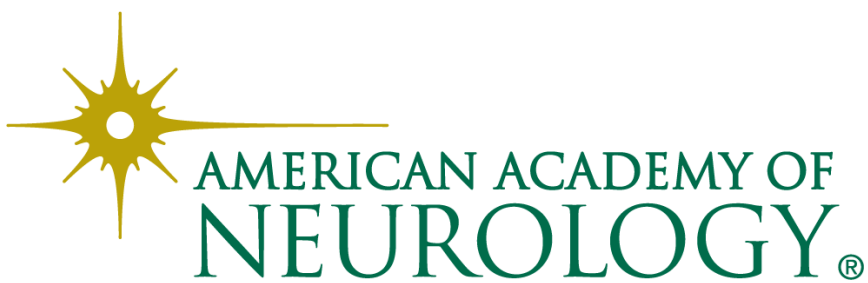

